G. BLUMENTHAL

Lick Observatory

University of California

Santa Cruz, CA 95064

USA

P. C. BOESHAAR

Department of Physics

Rider College

P.0. Box 6400

Lawrenceville, NJ 08648

USA

\section{A. BOSMA}

Observatoire de Marseille

2, Place Le Verrier

13248 Marseille Cédex 4

France

F . BOUCHET

IAP et École Polytechnique

98 bis, Boulevard Arago

75014 Paris

France

B. F. BURKE

26-335, Physics

MIT

Cambridge, MA 02139

USA

D. BURSTEIN

Department of Physics

Arizona State University

Tempe, AZ 85281

USA

R. H. BUSS, Jr.

Campus Box 391 APAS

University of Colorado

Boulder, C0 80309

USA

C. CANIZARES

37-501 Physics

MIT

Cambridge, MA 02139

USA
C. CARIGNAN

Kapteyn Laboratorium

Postbus 800

9700 AV Groningen

The Netherlands

R. CARLBERG

York University

4700 Keele Street

Downsview

Ontario M3J 1 P3

Canada

B. C. CARNEY

Department of Physics and Astronomy

Phillips Hall 039A

University of North Carolina

Chape1 Hi11, NC 27514

USA

B. CARR

School of Mathematical Sciences

Queen Mary College

Mile End Road

London E1 4NS

United Kingdom

S. CASERTANO

School of Natural Sciences

Institute for Advanced Study

Princeton, NJ 08540

USA

\section{A. CAULET}

Astronomy and Astrophysics

University of Chicago

5640 S. E11is Ave

Chicago, I1 60637

USA

\section{G. CHINCARINI}

Department of Physics and Astronomy University of Oklahoma

Norman, OK 73019

USA

M. CRÉZÉ

Observatoire de Besançon

41 Ave. de 1'Observatoire

25000 Besançon

France 


\author{
C. S. FRENK \\ Astronomy Centre \\ University of Sussex \\ Brighton BN1 9QH \\ United Kingdom \\ D. GERBAL \\ D.A.F., Observatoire de Meudon \\ 92195 Meudon Cédex \\ France
}

0. E. GERHARD

Max Planck Institut f. Astrophysik

Karl Schwarzschild Str. 1

D-8046 Garching bei München

Federal Republic of Germany

G. GILMORE

Institute of Astronomy

Madingley Road

Cambridge $\mathrm{CB} 3$ OHA

United Kingdom

J. H. VAN GORKOM

N.R.A.O., P.O. Box 0

Socorro, NM 87801

USA

J R. GOTT

Department of Astrophysical

Sciences

Princeton University

Princeton, NJ 08544

USA

R. F. GREEN

N.O.A.O.

P.0. Box 26732

Tucson, AZ 85726

USA

E. J. GROTH

Physics Department

Princeton University

Princeton, NJ 08544

USA

\author{
E. GUENDELMAN \\ Center for Theoretical Physics \\ MIT \\ Cambridge, MA 02139 \\ USA
}

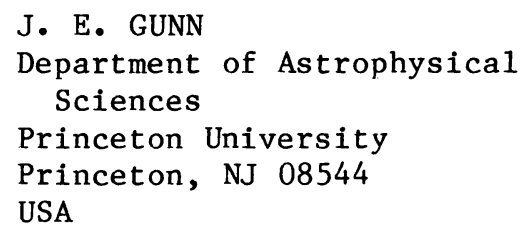

J. E. GUNN

Department of Astrophysical Sciences

Princeton University

Princeton, NJ 08544

USA
A. GUTH
Center for Theoretical Physics
MIT
Cambridge, MA 02139
USA

F. D. A. HARTWICK

University of Victoria

Department of Physics

P.0. Box 1700

Victoria, BC V8W $2 \mathrm{Y} 2$

Canada

J HEISLER

6-206 Physics

MIT

Cambridge, MA 02139

USA

L. HERNQUIST

Department of Astronomy

University of California

Berkeley, CA 94720

USA

J. HEWITT

26-349 Physics

MIT

Cambridge, MA 02139

USA

L. HOFFMAN

Department of Physics

Lafayette College

Easton, PA 18042

USA 
Y. HOFFMAN

Department of Physics

University of Pennsylvania

Philadelphia, PA 19104

USA

J. P. HUCHRA

Center for Astrophysics

60 Garden Street

Cambridge, MA 02138

USA

\section{P. HUT}

School of Natural Sciences

Institute for Advanced Study

Princeton, NJ 08540

USA

\section{S. IKEUCHI}

Tokyo Astronomical Observatory

Mitaka, Tokyo 181

Japan

G. ILLINGWORTH

Space Telescope Science Institute

Homewood Campus

Baltimore, MD 21218

USA

T. ISHIZAWA

Department of Astronomy

University of Kyoto

Kyoto 606

Japan

B. J. JARVIS

Cerro Tololo Interamerican Observatory

Casilla 603

La Serena

Chile

C. JOG

Department of Astronomy

P.0. Box 3818

University of Virginia

Charlottesville, VA 22903

USA
J. JONES

NORDITA

Blegdamsvej 17

DK-2100 K $\phi$ benhaven $\emptyset$

Denmark

B. JONES

NORDITA

Blegdamsvej 17

DK-2100 K $\varnothing$ benhaven $\emptyset$

Denmark

N. KAISER

Institute of Astronomy

Madingley Road

Cambridge $\mathrm{CB} 3$ OHA

United Kingdom

A. J. KALNAJS

Mt. Stromlo Observatory

Private Bag

Woden P.0., A.C.T.

2606 Australia

S. KENT

Center for Astrophysics

60 Garden Street

Cambridge, MA 02138

USA

G. R. KNAPP

Department of Astrophysical Sciences

Princeton University

Princeton, NJ 08544

USA

H. KODAMA

Department of Physics

University of Tokyo

Bunkyo-Ku

Tokyo 113

Japan

\section{J. KORMENDY}

Dominion Astrophysical Observatory

5071 W. Saanich Road

Victoria, BC V8X 4M6

Canada 
L. KRAUSS

Lyman Laboratory of Physics

Harvard University

Cambridge, MA 02138

USA

P. C. VAN DER KRUIT

Kapteyn Laboratorium

Postbus 800

$9700 \mathrm{AV}$ Groningen

The Netherlands

N. KRUMM

Physics Department

University of Cincinnati

Cincinnati, OH 45221-0011

USA

\section{LACEY}

Department of Astrophysical

Sciences

Princeton University

Princeton, NJ 08544

USA

M. LACHIÈZE-REY

Service d'Astrophysique

CEN-Saclay DPhG-SAp

91191 Gif-sur-Yvette Cédex

France

G. B. LAKE

School of Natural Sciences

Institute for Advanced Study

Princeton, NJ 08540

USA

R. B. LARSON

Astronomy Department

Yale University

New Haven, CT 06511

USA

D. LATHAM

Center for Astrophysics

60 Garden Street,

Cambridge, MA 02138

USA
B. LIEBERMAN

Center for Theoretical Physics

MIT

Cambridge, MA 02139

USA

F. J. LOW

Steward Observatory

University of Arizona

Tucson, AZ 85721

USA

V. N. LUKASH

Space Research Institute

USSR Academy of Sciences

Profsojusnaya 84-32

117810 Moscow

USSR

D. LYNDEN-BELL

Institute of Astronomy

Madingley Road

Cambridge CB3 OHA

United Kingdom

\section{J. MADSEN}

Institute of Astronomy

University of Aarhus

DK-8000 Aarhus C

Denmark

E. MALUMUTH

Department of Physics and Astronomy

Rutgers University

Piscataway, NJ 08854

USA

G. MAMON

Physics Department

New York University

4 Washington Place

New York, NY 10003

USA

L. MARTINET

Geneva Observatory

CH 1290 Sauverny

Switzerland 
R. D. MATHIEU

Center for Astrophysics

60 Garden St.

Cambridge, MA 02138

USA

J. MCCLINTOCK

37-521

Center for Space Research

MIT

Cambridge, MA 02139

USA

\section{J MCDOWALL \\ Institute of Astronomy \\ Madingley Road \\ Cambridge CB3 OHA \\ United Kingdom}

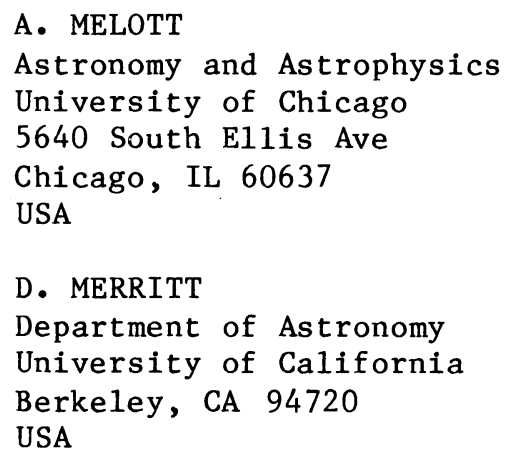

A. MELOTT

Astronomy and Astrophysics

University of Chicago

5640 South E11is Ave

Chicago, IL 60637

USA

D. MERRITT

Department of Astronomy

University of California

Berkeley, CA 94720

USA

M. MIJIC

452-48, Caltech

Pasadena, CA 91125

USA

\section{MILGROM}

Department of Nuclear Physics

The Weizmann Institute of Science

Rehovot 76100

Israel

J. MOODY

Institute for Theoretical Physics University of California

Santa Barbara, CA 93106

USA
R. A. NOLTHENIUS

Steward Observatory

University of Arizona

Tucson, AZ 85721

USA

C. A. NORMAN

Space Telescope Science Institute

Homewood Campus

Baltimore, MD 21218

USA

\section{F. OCCHIONERO}

Intituto Astronomico

University of Rome

Via G.M. Lancisi 29

00161 Rome

Italy

\section{J. P. OSTRIKER}

Department of Astrophysical Sciences

Princeton University

Princeton, NJ 08544

USA

\section{B. E. PACZYÑSKI}

Department of Astrophysical Sciences

Princeton University

Princeton, NJ 08544

USA

P. J. E. PEEBLES

Department of Physics

Princeton University

Princeton, NJ 08544

USA

R. C. PETERSON

Apt. D22

3636 North Campbe11

Tucson, AZ 85719

USA

J. PRIMACK

Physics Department

University of California

Santa Cruz

CA 95064

USA 
P. J. QUINN

130-33 Ca1tech

Pasadena, CA 91125

USA

\section{J. REES}

Institute of Astronomy

Madingley Road

Cambridge CB3 OHA

United Kingdom

D. O. RICHSTONE

Department of Astronomy

University of Michigan

Ann Arbor, MI 48109

USA

M. S. ROBERTS

NRAO

Edgemont Road

Charlot tesville

VA 22901

USA

H. J. ROOD

School of Natural Sciences

Institute for Advanced Study

Princeton, NJ 08540

USA

L. RUDNICK

School of Physics and Astronomy

University of Minnesota

116 Church St., SE

Minneapolis, MN 55455

USA

V. C. RUBIN

Department of Terrestrial Magnetism

Carnegie Inst. of Washington

5241 Broad Branch Road, NW

Washington, DC 20015

USA

E. E. SALPETER

Newman Laboratory of Natural Science

Cornel1 University

Ithaca, NY 14853

USA
R. SANCISI

Kapteyn Laboratorium

Postbus 800

9700 AV Groningen

The Netherlands

R. L. SANDERS

Kapteyn Laboratorium

Postbus 800

9700 AV Groningen

The Netherlands

C. L. SARAZIN

Department of Astronomy

P.0. Box 3818

University of Virginia

Charlottesville, VA 22903

USA

P. SCHECHTER

Mt. Wilson and Las Campanas Observatories

813 Santa Barbara St.

Pasadena, CA 91101

USA

M. SCHMIDT

105-24 Astronomy

Ca1tech

Pasadena, CA 91125

USA

M. SCHWARZSCHILD

Department of Astrophysical Sciences

Princeton University

Princeton, NJ 08544

USA

D. SECKEL

Fermilab, MS 209

P.0. Box 500

Batavia, IL 60510

USA

P. SEITZER

N.0.A.0., P.0. Box 26732

Tucson, AZ 85726

USA 
P.A. THOMAS

Institute of Astronomy

Madingley Road

Cambridge CB3 OHA

United Kingdom

S. D. TREMAINE

C.I.T.A.

McLennan Physical Lab.

University of Toronto

60 St. George Street

Toronto, Ontario M5S $1 \mathrm{Al}$

Canada

G. TRINCHIERI

Center for Astrophysics

60 Garden Street

Cambridge, MA 02138

USA

W. TUCKER

Center for Astrophysics

60 Garden Street

Cambridge, MA 02138

USA

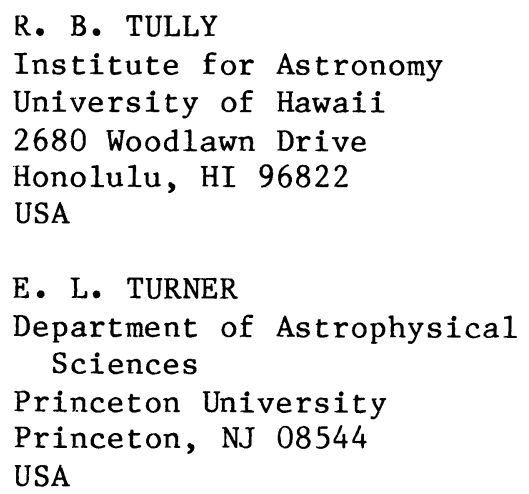

M. S. TURNER

Fermilab, MS 209

P.0. Box 500

Batavia, IL 60510

USA

J. A. TYSON

AT\&T Bel1 Laboratories

ID-316, Murray Hi11

NJ 07974

USA

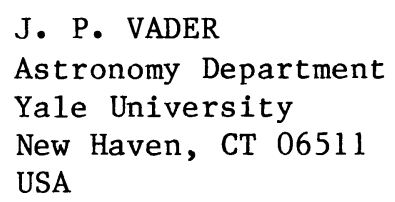

S. VEERARAGHAVAN

Department of Astronomy

University of California

Berkeley, CA 94720

USA

E. T. VISHNIAC

Astronomy Department

University of Texas

Austin, TX 78712

USA

W. D. WATSON

Loomis laboratory of Physics

University of Illinois

1110 W. Green Street

Urbana, IL 61801

USA

S. D. M. WHITE

Steward Observatory

University of Arizona

Tucson, AZ 85721

USA

B. WHITMORE

Space Telescope Science Institute Homewood Campus

Baltimore, MD 21218

USA

D. WILKINSON

Department of Physics

Princeton University

Princeton, NJ 08544

USA

T. B. WILLIAMS

Department of Physics and Astronomy

Rutgers University

Piscataway, NJ 08854

USA 


\section{H. VAN WOERDEN}

Kapteyn Laboratorium

Postbus 800

9700 AV Groningen

The Netherlands

\section{J. P. WRIGHT}

National Science Foundation

Extragalactic Astronomy

Washington, DC 20550

USA

\section{W. WUENSCH}

Department of Physics and Astronomy

University of Rochester

Rochester, NY 14627

USA

\section{R. WYSE}

Department of Astronomy

University of California

Berkeley, CA 94720

USA

A. YAHIL

Astronomy Program

State University of New York

Stony Brook, NY 11794

USA

W. H. ZUREK

Theoretical Astrophysics

T-6, MS B288

LANL, Los Alamos

NM 87545

USA 